\title{
Do elderly patients benefit from implantable defibrillators?
}

“...in addition to meeting traditional eligibility criteria, the decision to implant an implantable cardioverter defibrillator in the elderly should also be individualized, focusing on comorbidities, projected quality of life and patient preference."

The advent of implantable cardioverter defibrillators (ICDs) has revolutionized the prevention of sudden arrhythmic death in patients with severe left ventricular systolic dysfunction, with or without concomitant heart failure, and selected patients with a prior history of sustained ventricular arrhythmias. Supported by large multicenter randomized trials, there has been a widespread response of ICD implantation in developed countries for patients who meet consensus guidelines [1]. However, the guidelines do not provide guidance when considering device implantation in elderly patients. With the growing number of elderly persons being evaluated by cardiologists, the potential implications of greater expenditure on prophylactic devices must be weighed against the implicit rationing based on age. These factors are counter-balanced by the sometimes counterdirectional priorities proposed by placing greater value on quality versus quantity of life.

Although elderly patients were not systematically excluded from randomized trials, they were vastly under-represented and those who were included had few comorbidities by virtue of the strict inclusion criteria intrinsic to trial design. Pooled analyses of the randomized trials suggested that the benefit from ICD implantation in elderly patients may be diminished $[2,3]$. In order to better understand the natural history of elderly ICD recipients, previously published observational studies have endeavored to be more inclusive and representative of real-life patients and outcomes. However, these studies have been restricted by small sample size, insufficient clinical information to adequately control for confounders, and/or limited breadth and duration of follow-up [4-8]. As such, we recently published one of the largest and most expansive indication- and age-stratified analyses of ICD implantation to date, based on our experience in Ontario (Canada) [9].

The Ontario ICD Registry is a large, prospective database designed to evaluate adjudicated clinical and device-related outcomes of patients implanted with an ICD [10]. The study was unique since enrollment in the registry was mandatory for all patients who underwent ICD implantation in the province, excluding the possibility of participation bias [9]. Among 3939 primary and 1460 secondary prevention ICD recipients between 2007 and 2010, overall, mortality increased with age, grouped into the following categories: $18-49,50-59,60-69$, $70-79$ and $\geq 80$ years. Appropriate shocks were not significantly different across age groups, with an overall average of 5.1 and 12.0 events per 100 person-years for primary and secondary prevention cohorts, respectively. However, there was a slight downward trend with older age groups. Furthermore, the rates of 45-day periprocedural complications and inappropriate shocks were also similar across age groups. Mortality rates were highest albeit relatively modest among octogenarians at 10.2 and 15.5 deaths per 100 person-years for primary and secondary prevention ICD recipients, respectively [9]. Despite these encouraging findings, there are several caveats in the interpretation of our study.

It would be overly simplistic to suggest that the findings of our study imply that all patients should be implanted with ICDs, irrespective of their age. While it may seem counterintuitive, our data do not support that all elderly patients who meet guideline criteria should be implanted with the device. Indeed, we found that elderly patients who were implanted with ICDs in Ontario had fewer comorbidities than expected for their age and a lower underlying degree of cardiac disease or dysfunction. The selective nature of determining ICD candidacy, irrespective of age, may have been attributed to the uniform training of cardiac electrophysiologists at high-volume centers who are the gatekeepers of device implantation in Ontario. Furthermore, patients were well treated with high rates of evidence-based medical therapies
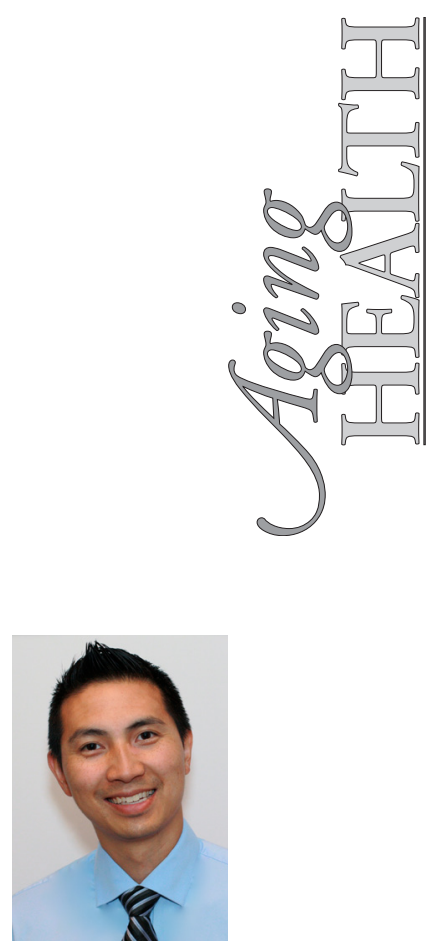

\section{Derek Yung}

Institute for Clinical Evaluative Sciences, University of Toronto, Room G-106, 2075 Bayview Avenue Toronto, ON, M4N 3M5, Canada

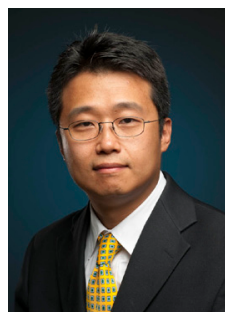

Douglas S Lee Author for correspondence: Institute for Clinical Evaluative Sciences, University of Toronto, Room G-106, 2075 Bayview Avenue Toronto, ON, M4N 3M5, Canada Fax: +14164806048 dlee@ices.on.ca

\section{Keywords}

- age $\bullet$ benefit $\bullet$ death $\bullet$ elderly

- implantable defibrillators

- prognosis • risk • survival 
before decisions to implant the ICD were rendered, attesting to the relative robustness and high quality of care provided to implantable defibrillator candidates in Ontario.

“...the major impediment to implantable cardioverter defibrillator efficacy is the increasing proportional risk of nonarrhythmic death..."

An indication that the ICD delivered electrical treatment for a potentially life-threatening arrhythmia (i.e., ventricular tachycardia or fibrillation) is the occurrence of a defibrillator shock. We found that despite the higher mortality of elderly ICD recipients, the rates of appropriate device shock were not significantly lower than those among younger patients. Since ICD shocks are highly efficient in preventing sudden death, it may seem reasonable to infer that if elderly patients do not exhibit significantly lower rates of appropriate device shocks, we could conclude that ICDs improve survival in the elderly. However, this hypothesis was not tested in our recently published study [9]. A direct correlation between preserved rates of ICD shock and mortality benefit cannot be conclusively known as an appropriate shock is not necessarily equivalent to an aborted sudden arrhythmic death event [11]. Furthermore, appropriate treatment of ventricular arrhythmias and reduction in sudden arrhythmic death may not result in beneficial effects on total mortality $[12,13]$.

Irrespective of age, the major impediment to ICD efficacy is the increasing proportional risk of nonarrhythmic death, which can blunt and potentially abrogate the benefit of ICD therapy $[7,14]$. Appropriate ICD shocks and death are competing events since early death reduces the likelihood of receiving an appropriate therapy from the device. Analyses that do not account for competing risks may inappropriately suggest that factors that are associated with shorter survival may also reduce the probability of receiving an appropriate ICD shock. Our report was novel because we used a competing risks analytical framework to examine the impact of age. We found that the competing risk of death inflected upwards at 60-69 years for primary and $\geq 80$ years for secondary prevention ICD recipients, suggesting a differential impact of age among primary and secondary prevention patients [9]. This observation highlights the clinically distinct nature of the patient populations undergoing ICD implantation for primary prophylaxis versus secondary prevention, which are defined by the occurrence of cardiac arrest or symptomatic ventricular tachyarrhythmia prior to device implantation. Despite these potential differences, the robustness of our findings of preserved rates of appropriate ICD shock across all age groups was demonstrated.

Quality of life may be an increasingly important priority for elderly individuals, encompassing physical and psychological wellbeing. In the population-based Ontario ICD Registry, we also noted that all referred patients in Ontario were considered for implantation regardless of age, and the most common reason for nonimplantation in qualified candidates was patient refusal, which occurred twice as often in octogenarians [9]. This underscores the importance of discussing anticipated benefits from the ICD in relation to functional capacity and patient preference. At the most basic level, this entails a discussion of prolongation of life versus the need for invasive procedures, including the initial device implant, lead revisions and generator replacements when devices are at the end of battery life. It also entails a discussion about living with the possibility of inappropriate device shocks, which may be symptomatically quite painful.

"...the population is aging and the dilemma of implantable cardioverter defibrillator candidacy in the elderly will continue to resonate."

The prognostic implications of appropriate device shocks are also not completely defined, but they may differ in the elderly compared with younger patients. Our analysis demonstrated that the prognostic impact of ICD shocks was more pronounced among the elderly than younger individuals, manifested by higher postshock mortality rates among elderly patients [9]. The reasons for this finding were beyond the scope of our report, but it may suggest that a shock in older individuals relates to underlying cardiac disease progression or a worsening noncardiac comorbidity profile. By contrast, among younger patients, the relatively lower mortality rates after an appropriate ICD shock may be more indicative of a transient cause of ventricular tachyarrhythmia or one that is more easily amenable to treatment.

Since age alone is insufficient as a marker to guide clinicians who are deciding ICD candidacy, there is a need for further research to better risk-stratify patients who meet guideline criteria for defibrillator implantation. Although 
advanced imaging modalities and electrophysiologic techniques are being increasingly proposed for ICD risk stratification, at the most fundamental level, simple clinical risk predictors require further examination to determine their utility in decision support. As an example, our examination of sex and ICD outcomes demonstrated differential rates of device-delivered appropriate therapies that were lower in women compared with men $[10]$. Other important clinical predictors of outcome merit consideration in a multivariate risk model that could be used to further enhance decision-making and discussions about the risks and benefits of ICD implantation, with elderly patients who are potential candidates for ICDs.

In summary, the population is aging and the dilemma of ICD candidacy in the elderly will continue to resonate. Many countries have been faced with the convergence of a growing elderly population and limited availability of healthcare resources. At a population level, the role of the treating physician in judicious resource allocation is vital. Our study provides guidance to physicians and patients by demonstrating that age alone should not preclude ICD implantation.
However, in addition to meeting traditional eligibility criteria, the decision to implant an ICD in the elderly should also be individualized, focusing on comorbidities, projected quality of life and patient preference.

\section{Disclaimer}

The opinions, results and conclusions are those of the authors and no endorsement by the Ministry of Health and LongTerm Care or by the Institute for Clinical Evaluative Sciences is intended or should be inferred.

\section{Financial \& competing interests disclosure}

The Institute for Clinical Evaluative Sciences (ICES) is supported in part by a grant from the Ontario Ministry of Health and Long-Term Care. This research was supported by an operating grant from the Canadian Institutes of Health Research (CIHR MOP 111150) and the Ontario Ministry of Health and Long-Term Care. DS Lee is a clinician-scientist of the CIHR. The authors have no other relevant affiliations or financial involvement with any organization or entity with a financial interest in or financial conflict with the subject matter or materials discussed in the manuscript apart from those disclosed.

No writing assistance was utilized in the production of this manuscript.

\section{References}

1. Epstein AE, Di Marco JP, Ellenbogen KA et al:; American College of Cardiology/ American Heart Association Task Force on Practice Guidelines (Writing Committee to Revise the ACC/AHA/NASPE 2002 Guideline Update for Implantation of Cardiac Pacemakers and Antiarrhythmia Devices); American Association for Thoracic Surgery; Society of Thoracic Surgeons. ACC/AHA/HRS 2008 guidelines for device-based therapy of cardiac rhythm abnormalities: a report of the American College of Cardiology/American Heart Association Task Force on practice guidelines (Writing Committee to revise the ACC/AHA/NASPE 2002 guideline update for implantation of cardiac pacemakers and antiarrhythmia devices): developed in collaboration with the American Association for Thoracic Surgery and Society of Thoracic Surgeons. Circulation 117(21), e350-e408 (2008).

2. Healey JS, Hallstrom AP, Kuck KH et al. Role of the implantable defibrillator among elderly patients with a history of life-threatening ventricular arrhythmias. Eur. Heart J. 28(14), 1746-1749 (2007).

3. Santangeli P, Di BL, Dello RA et al. Meta-analysis: age and effectiveness of prophylactic implantable cardioverterdefibrillators. Ann. Intern. Med. 153(9), 592-599 (2010).

4. Chan PS, Nallamothu BK, Spertus JA et al. Impact of age and medical comorbidity on the effectiveness of implantable cardioverterdefibrillators for primary prevention. Circ. Cardiovasc. Qual. Outcomes 2(1), 16-24 (2009).

5. Epstein AE, Kay GN, Plumb VJ et al. Implantable cardioverter-defibrillator prescription in the elderly. Heart Rhythm 6(8), 1136-1143 (2009).

6. Koplan BA, Epstein LM, Albert CM, Stevenson WG. Survival in octogenarians receiving implantable defibrillators. Am. Heart J. 152(4), 714-719 (2006).

7. Lee DS, Tu JV, Austin PC et al. Effect of cardiac and noncardiac conditions on survival after defibrillator implantation. J. Am. Coll. Cardiol. 49(25), 2408-2415 (2007).

8. Tsai V, Goldstein MK, Hsia HH, Wang Y, Curtis J, Heidenreich PA. Age differences in primary prevention implantable cardioverter-defibrillator use in US individuals. J. Am. Geriatr. Soc. 59(9), 1589-1595 (2011).

9. Yung D, Birnie D, Dorian P et al. Survival after implantable cardioverter-defibrillator implantation in the elderly. Circulation 127(24), 2383-2392 (2013).

10. MacFadden DR, Crystal E, Krahn AD et al. Sex differences in implantable cardioverter-defibrillator outcomes: findings from a prospective defibrillator database. Ann. Intern. Med. 156(3), 195-203 (2012).

11. Germano JJ, Reynolds M, Essebag V, Josephson ME. Frequency and causes of implantable cardioverter-defibrillator therapies: is device therapy proarrhythmic? Am. J. Cardiol. 97(8), 1255-1261 (2006).

12. Hohnloser SH, Kuck KH, Dorian P et al. Prophylactic use of an implantable cardioverter-defibrillator after acute myocardial infarction. N. Engl. J. Med. 351(24), 2481-2488 (2004).

13. Bigger JT. Prophylactic use of implanted cardiac defibrillators in patients at high risk for ventricular arrhythmias after coronary-artery bypass graft surgery. Coronary Artery Bypass Graft (CABG) Patch Trial Investigators. N. Engl. J. Med. 337(22), 1569-1575 (1997).

14. Krahn AD, Connolly SJ, Roberts RS, Gent M. Diminishing proportional risk of sudden death with advancing age: implications for prevention of sudden death. Am. Heart J. 147(5), 837-840 (2004). 